\title{
Using High-Resolution Digital Aerial Imagery To Map Land Cover
}

The Upper Midwest Environmental Sciences Center (UMESC) has used aerial photography to map land cover/land use on federally owned and managed lands for over 20 years. Until recently, that process used 23- by 23-centimeter (9- by 9-inch) analog aerial photos to classify vegetation along the Upper Mississippi River System, on National Wildlife Refuges, and in National Parks. With digital aerial cameras becoming more common and offering distinct advantages over analog film, UMESC transitioned to an entirely digital mapping process in 2009 . Though not without challenges, this method has proven to be much more accurate and efficient when compared to the analog process.
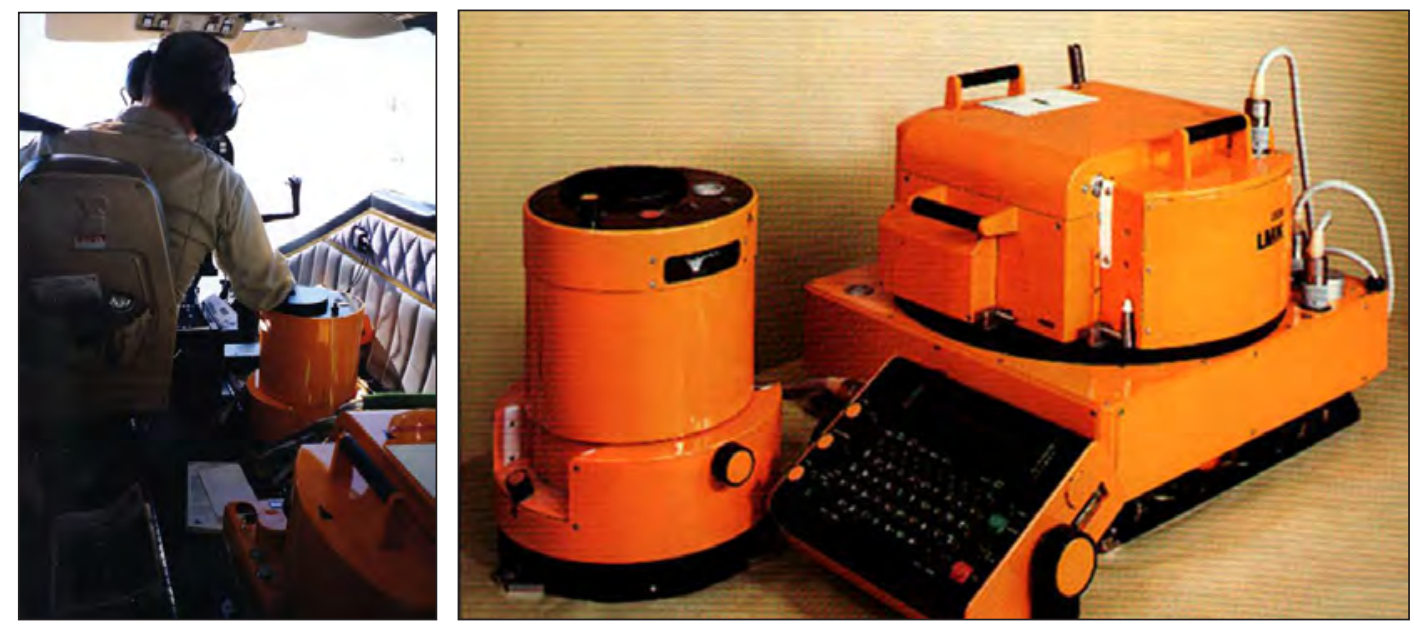

Figure 1. The 23- by 23-centimeter (9- by 9 -inch) aerial camera with a 152.4-millimeter (6-inch) lens has been the primary high-resolution delivery system for documenting the Nation's natural resources.
The U.S. Geological Survey's mission is to provide reliable scientific information that describes and helps us better understand our Earth. From the beginning, remote sensing, and aerial photography in particular, have played crucial roles in the monitoring and management of our natural resources. The Federal government has been collecting aerial photography of the Nation's trust lands for almost 100 years (W.N. Brown, Inc., 1930).

The Upper Midwest Environmental Sciences Center (UMESC), collaborating with the U.S. Army Corps of Engineers and the U.S. Fish and Wildlife Service (USFWS), has collected or acquired aerial imagery from the 1920s up to the present date. For most of that time period aerial photography has been collected with a 23- by 23-centimeter (9- by 9-inch, also called 9X9) aerial camera like the one pictured in figure 1. Not only is this format mature and reliable, but so are the geographic information system (GIS) software and hardware that support 9X9 photography. Individual frames can be scanned by photogrammetric scanners at 10 to 20 micrometers $(1,270-2,540$ dots per inch, or dpi), capturing an extraordinary amount of detail for photography flown at larger scales. Despite its maturity and reliability, however, this system can also be inefficient and inflexibile. The drawbacks include limited film payload, no real-time feedback on image quality, limited exposure information, and specialized handling of the aerial film (cold storage, loading and unloading in complete darkness, chemical-based processing, and scanning for use in GIS programs).

\section{The Digital Transition}

Around the turn of the 21 st century, and with the digital revolution in full swing, some aerial photographers began using commercial digital single lens reflex (SLR) cameras for aerial imagery (Mills and others, 1996). These cameras could be either strapped to the plane's struts or wings and fired remotely or pointed through belly hatches and fired directly by the user. Figure 2 illustrates how UMESC documented waterfowl habitat use by mounting a Kodak DCS Pro $14 n^{1}$ camera in the USFWS Region 3 Partenavia plane and using it to collect vertical digtal imagery of waterfowl from 2003 to 2006. Although the Kodak camera had one of the largest digital SLR sensors of the day at 14 megapixels (MP), it could collect imagery only in natural color, not the color infrared (CIR) necessary for most vegetation analyses. Referencing the imagery to the Earth's surface (also called georeferencing) and mosaicking efforts were cumbersome and resulted in inconsistent accuracy, but it was clear nonetheless that digital aerial acquisition had the potential to be revolutionary. Image thumbnails on the camera back provided instant exposure assessment, and automated image processing allowed for rapid turnaround. Aerial camera manufacturers were also taking note of this potential and began adapting, then enhancing the professional digital SLR camera sensors for aerial use. Soon digital cameras were designed as complete systems

${ }^{1}$ Any use of trade, firm, or product names is for descriptive purposes only and does not imply endorsement by the U.S. Government. 


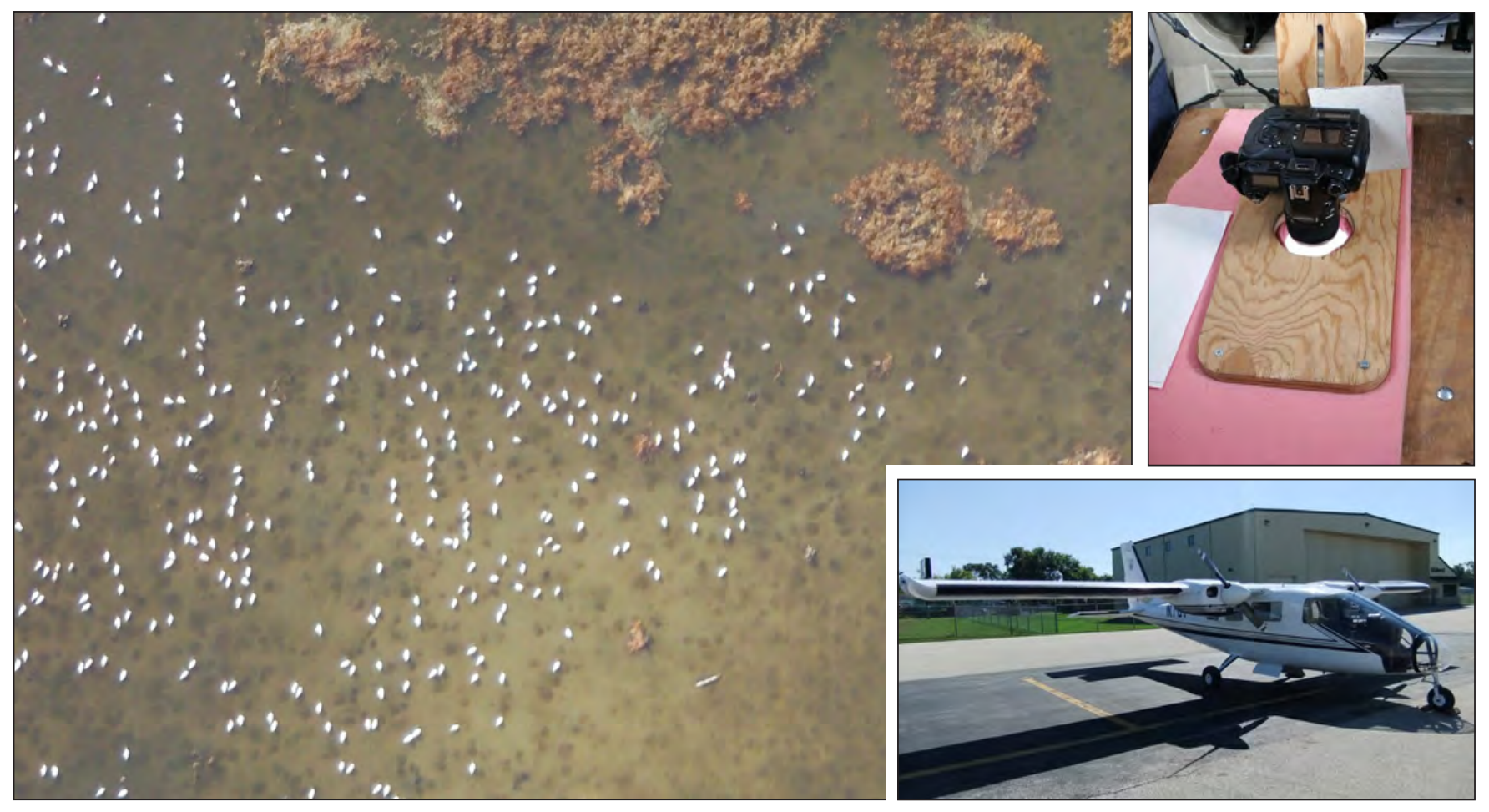

Figure 2. A digital camera with a wide-angle zoom lens mounted in the belly hatch of a plane collects vertical digital imagery of tunrda swan habitat use on the Upper Mississippi River. The swans (small white dots) are feeding on aquatic plant tubers, as evidenced by the darker "pitting" visible in shallow water's muddy substrate.

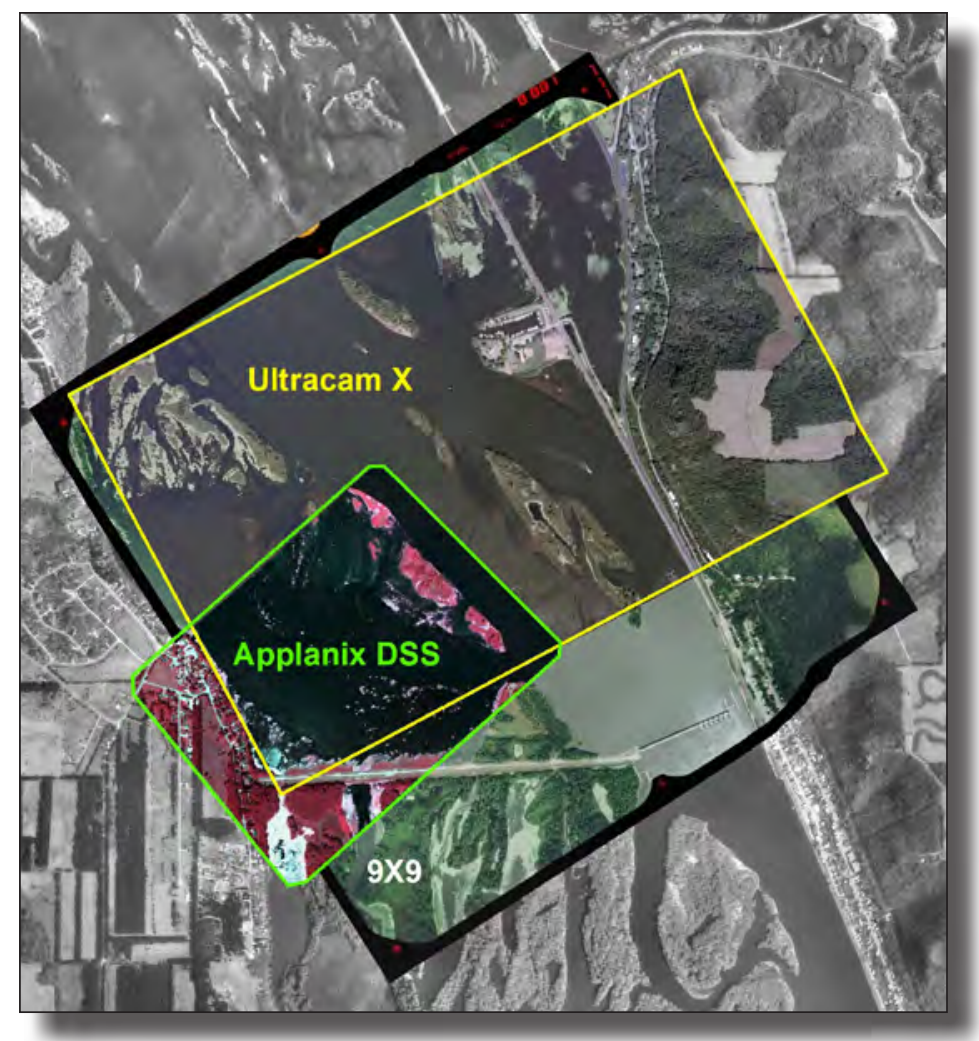

\section{Image Metrics}

- Ultracam X: $14,430 \times 9,420$ pixels at 20 centimeters (8 inches)/pixel

- Applanix DSS 439: 7,216 × 5,412 pixels at 20 centim/pixel

- 9X9: 7,200 × 7,200 pixels at 41 centimeters (16 inches)/pixel based on a 32-micrometer (800-dpi) scan size.

Figure 3. A comparison of the extents of a 1:12,000-scale 23- by 23-centimeter ( 9 - by 9 -inch) aerial photo, a 216-megapixel largeformat digital aerial camera (Ultracam X), and a 39-megapixel medium-format digital aerial camera (Applanix DSS 439), the latter two collected at 20 centimeters ( 8 inches)/pixel. Photo location is Lock and Dam 4 on the Upper Mississippi River. 
for aerial imagery acquisition and quickly began replacing their analog film counterparts.

It was clear from UMESC's use of the Kodak SLR that digital photography could be used to assess the landscape, and in 2006 UMESC contracted test imagery acquisition for Navigation Pools 6 and 8 of the Upper Mississippi River using a 4-band, 86-MP Ultracam D and compared it directly with scanned 9X9 analog film of the same areas. The digital imagery showed better contrast and color and, when resampled to the same resolution as the scanned imagery, had more detail than the scanned film. Another test of the same areas in 2007 confirmed the usefulness of digital aerial imagery for vegetation mapping. In October 2009, this decision was affirmed yet again when Kodak announced that it was discontinuing production of its 23-centimeter CIR aerial film, KODAK AEROCHROME III Infrared Film 1443, owing to decreasing demand (Kodak, 2009).

Although the first mapping-grade sensors were fairly small by current standards, between 6 and $16 \mathrm{MP}$, technological advances in camera electronics and processors have allowed sensors to add more pixels and collect much larger images much faster. Currently (December 2013), the largest framebased and pushbroom sensors widely available for purchase are 20,000 pixels wide. Microsoft's newest digital mapping camera, the 260-MP Ultracam Eagle, collects frame-based images at 20,010 by 13,080 pixels, equivalent to a 9 - by 6 -inch filmbased photo scanned at 12 micrometers (2,117 dpi) (Microsoft, 2013). By using an 80-millimeter (3.15-inch) lens at an altitude of 1,525 meters $(5,000$ feet $)$ above ground level, this would be the equivalent to a 1:19,000-scale aerial photo with a scanned resolution of 23 centimeters per pixel. Scientists and resource managers who have always thought of aerial photography in terms of scale typically used for 9X9 film photography will have to recalibrate their minds and metrics to image resolution and pixels associated with digital imagery. Figure 3 shows a comparison between an Ultracam X frame, an Applanix DSS frame, and a $9 \times 9$ aerial.

Digital aerial imagery has many other advantages over film for resource mapping. As mentioned previously, thumbnail images are displayed on the camera system's monitor, providing immediate exposure feedback. Many cameras are able to collect four bands (red, green, blue, and near infrared) within the same image, which allows the user to display the image as either natural color or CIR (fig. 4). The digital sensors are capable of collecting 12-bit or higher data, increasing the amount of data stored per pixel and ensuring useful imagery in a wide variety of lighting conditions. For each mission a log is maintained that precisely documents, at exposure, the camera's horizontal and vertical positions by using a global positioning system and the camera sensor's pitch, roll, and crab as determined by an inertial measurement unit. Some of the measurements collected at exposure are listed below.

\begin{tabular}{|c|c|}
\hline Time & Omega $^{* *}$ \\
\hline Image number & $\mathrm{Phi}^{* *}$ \\
\hline Flight line number & Kарpa $^{* *}$ \\
\hline Latitude & Shutter speed \\
\hline Longitude & Exposure compensation \\
\hline Altitude & Aircraft speed \\
\hline Pitch $^{*}$ & Northing \\
\hline Roll* & Easting \\
\hline Heading* & Geoid height \\
\hline
\end{tabular}

\section{Image Display and Analysis}

Aerial imagery intended for interpretation is always collected in stereo, where each frame overlaps the adjacent frame by approximately 60 percent. This allows the same scene to be viewed from two slightly different perspectives and provides the illusion of depth in a manner similar to how our eyes see the world. In order to view these images in stereo, they need to be precisely aligned. Because digital images can no longer be viewed on stereoscopes designed for analog film, they must be viewed on 3D computer monitors. Specialized GIS software imports image metadata and uses it to generate stereo models and georeferenced image mosaics. When combined with 3D computer hardware (3D monitor, 3D glasses, and a high-end
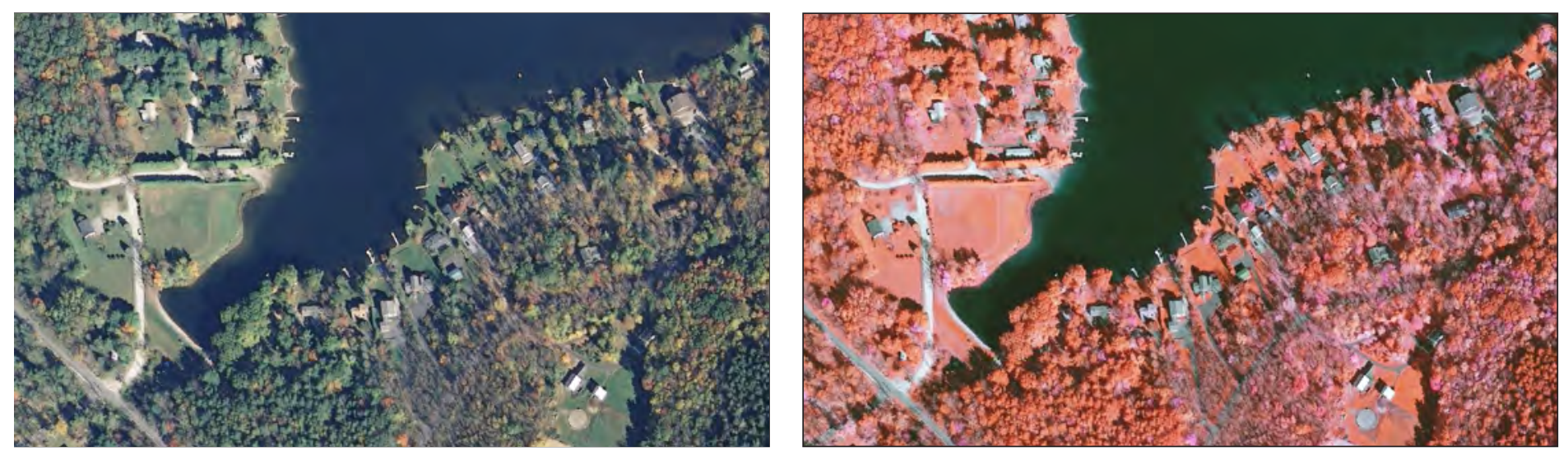

Figure 4. A four-band digital image can be displayed in most GIS programs as an RGB (red/green/blue) image or as CIR (color infrared) image. In the CIR image, the more chlorophyll present in the vegetation, the redder it appears. 

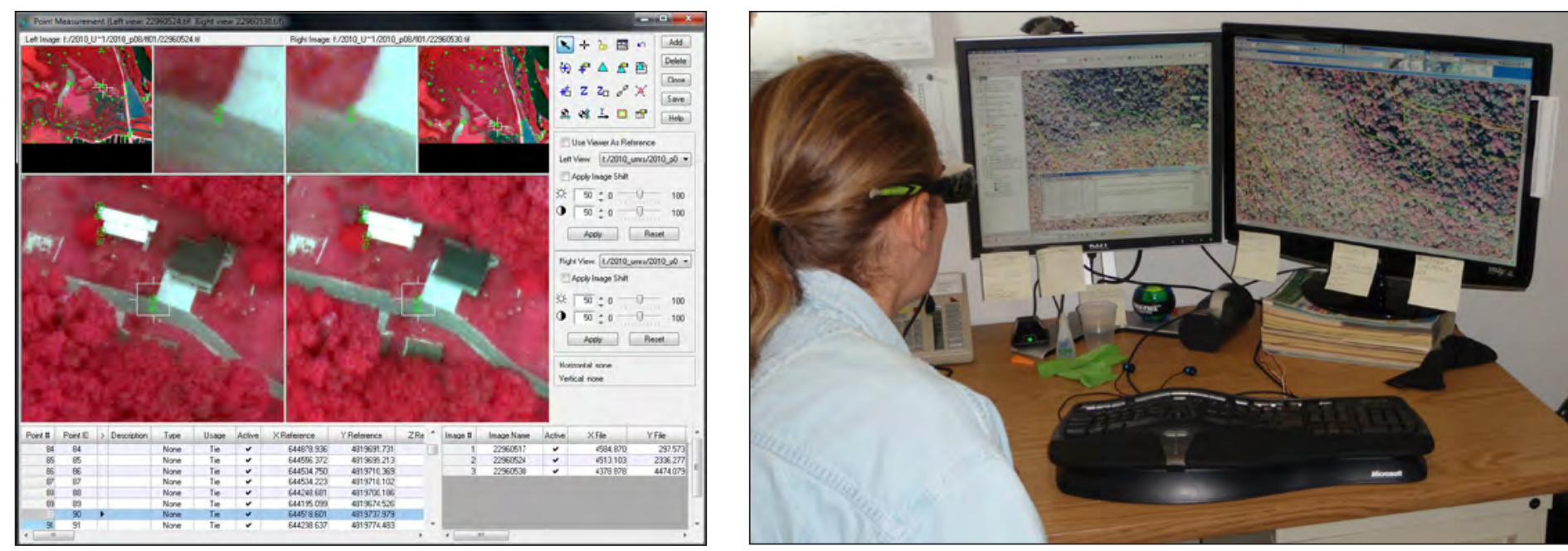

Figure 5. Software applications are used to process raw digital image data into GIS-ready stereo images, then synchronize 3D glasses and monitors to deliver a bright, stable stereo image.

graphics card), this software allows the user to view and delineate vegetation in high-resolution 3D by means of a computer mouse (fig. 5). Because the stereo images are already georeferenced to the Earth, all delineations are also georeferenced, providing real-time position and area information.

The transition from analog aerial photography to digital for resource monitoring and vegetation mapping has been very successful for UMESC and its partners. Many previously tedious and time-consuming tasks, such as image orthorectification, are now largely automated and much more accurate. As a result, land-cover and land-use mapping is faster and more accurate.

UMESC and its partners continue to collaborate on the collection and use of aerial imagery in support of natural resource management. Planned assessments include development of thermal infrared orthomosaics for detection of waterfowl, deer, and the cold air vents associated with algific talus slopes (home to the endangered Iowa Pleistocene snail and threatened Northern monkshood). UMESC is also assessing terrain extraction software that uses aerial imagery to generate high-resolution digital surface and elevation models for use in GIS applications requiring precise height information, such as water level models and forest maturity assessments. Digital imagery is not without its own disadvantages: startup and maintenance costs can be very high, and state-of-the-art technology can become obsolete within a few years as camera and sensor capabilities continue to evolve. However, its advantages far outweigh these drawbacks, and UMESC intends to remain on the cutting edge in the use of high-resolution digital aerial imagery for resource monitoring and mapping.

\section{References Cited}

Kodak, 2009, KODAK AEROCHROME III Infrared Film 1443: Web page, accessed April 8, 2014, at http://www.kodak. com/eknec/PageQuerier.jhtml?pq-path $=4011 / 4013 \&$ pqlocale $=e n$.

Microsoft, 2013, Ultracam Eagle specifications: Web page, accessed April 8, 2014, at http://www.microsoft.com/ ultracam/en-us/UltraCamEagleSpecs.aspx.

Mills, J.P., Newton, I., and Graham, R.W., 1996. Aerial photography for survey purposes with a high resolution, small format, digital camera: Photogrammetric Record, v. 15, no. 88 , p. 575-587.

W.N. Brown, Inc., 1930, Upper Mississippi River, Hastings, Minnesota to Grafton, Illinois - Survey 1929-1930: Washington, D.C., Williams \& Heintz Co., prepared under contract for U.S. Engineer Office, 121 maps.

\section{For more information on UMESC's use of aerial imagery, please contact:}

Jennifer Dieck

jdieck@usgs.gov

608-781-6382
Larry Robinson

lrobinson@usgs.gov

608-781-6354

U.S. Geological Survey, 